\title{
Hypoplastic Breast Anomalies in the Female Adolescent Breast
}

\author{
Sebastian Winocour, MD, MSc ${ }^{1}$ Valerie Lemaine, MD, MPH, FRCSC ${ }^{1}$ \\ ${ }^{1}$ Division of Plastic Surgery, Department of Surgery, Mayo Clinic, \\ Rochester, Minnesota \\ Semin Plast Surg 2013;27:42-48. \\ Address for correspondence Valerie Lemaine, MD, MPH, FRCSC, \\ Assistant Professor of Plastic Surgery, Vice Chair for Research, Department \\ of Surgery, Division of Plastic Surgery, Mayo Clinic, 200 First Street SW, \\ Rochester, MN 55905 (e-mail: Lemaine.valerie@mayo.edu).
}

\begin{abstract}
Hypoplastic breast anomalies remain a reconstructive challenge. In this article, the authors review the spectrum of nonsyndromic breast hypoplasia in the adolescent girl, namely idiopathic breast hypoplasia and tuberous breast deformity. Challenges specific to this population include (1) identifying the ideal timing for surgery, (2) choosing the optimal reconstructive method while taking into consideration ongoing changes in the

Keywords

- breast hypoplasia

- tuberous breast

- adolescent female body (e.g., future pregnancy), and (3) considering the use of a two-stage reconstructive approach to gradually expand the skin envelope. A comprehensive review of surgical techniques that address these conditions is provided, taking into account the evolution and the most recent advances in surgical techniques.
\end{abstract}

\section{Idiopathic Breast Hypoplasia}

Breast hypoplasia in the adolescent girl remains a reconstructive challenge ( $\boldsymbol{- F i g . 1}$ ). Understanding the etiology of this deformity is fundamental to achieving best outcomes. Breast hypoplasia may be congenital or acquired. The defect can be isolated or associated with other pathology, including syndromes and chest wall anomalies. Examples of associated hypoplastic syndromes are Jeune and Poland syndromes. Chest wall deformities include pectus excavatum and pectus carinatum. In this article, we will address the diagnosis and management of breast hypoplasia secondary to abnormal adolescent development; breast hypoplasia associated with congenital syndromes and chest wall deformities will be discussed elsewhere in this publication.

Breast hypoplasia in the adolescent girl can either be unilateral or bilateral. Unilateral hypoplasia is present when there is significant discrepancy in mammary size between breasts and there is no evidence of macromastia of the contralateral breast. ${ }^{1}$ Bilateral breast hypoplasia may be either symmetric or asymmetric. ${ }^{2}$ Histologically, both unilateral and bilateral hypoplastic breast tissue consist of fibrous stroma and ductal structures without acinar differentiation. Ducts typically resemble those in the prepubertal breast and lack lobular development. ${ }^{1}$

Issue Theme The Adolescent Breast; Guest Editors, Valerie Lemaine, MD, MPH, FRCSC and Patricia S. Simmons, MD
Most cases of nonsyndromic breast hypoplasia are idiopathic, but some have an identifiable cause. Acquired mammary hypoplasia has been reported in patients who have received breast radiation in infancy or childhood, most frequently for the treatment of cutaneous hemangiomas. Studies have demonstrated that the degree of hypoplasia directly correlates with the amount of radiation administered to the patient. ${ }^{1}$ Other iatrogenic causes include previous tube thoracostomy, thoracotomy, and excision of both benign and malignant breast tumors during childhood leading to impaired breast development. ${ }^{3}$ Burns to the anterior chest can lead to failure of complete mammary expansion from injury to the breast bud. ${ }^{4}$ Medication-induced breast hypoplasia has also been described. ${ }^{2}$ Finally, there has been one reported case of unilateral breast atrophy following normal breast development from infectious mononucleosis in an adolescent girl. $^{5}$

Breast hypoplasia carries a significant psychological burden, especially in the adolescent population. The young may suffer from low self-esteem, depression, social anxiety, peer rejection, and psychosexual dysfunction. These problems can lead to significantly impaired psychosocial development. ${ }^{4,6}$ This impact has become a major factor in defining the timing of surgical intervention in these patients.

There are conflicting views in the literature and practice regarding the optimal timing for surgical intervention in
$10.1055 / \mathrm{s}-0033-1343$
ISSN $1535-2188$.

ISSN 1535-2188. 


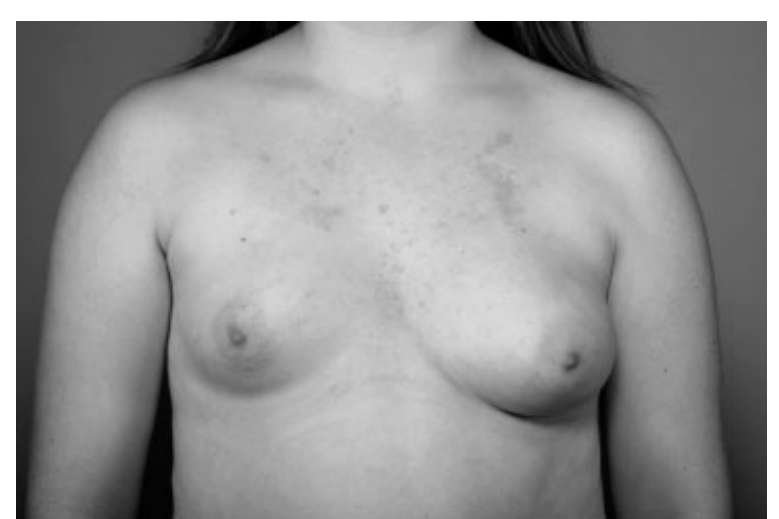

Fig. 1 Idiopathic breast hypoplasia in an adolescent girl.

breast hypoplasia in adolescence. Historically, many have recommended that treatment be deferred until breast development is complete, defined as having reached Tanner stage $\mathrm{V}$, and the patient has maintained a stable adult weight and breast volume for one year. ${ }^{6,7}$ This minimizes the risk of asymmetrical growth and development that may otherwise necessitate additional surgery. Furthermore, it eliminates the risk of surgical site infection that may be associated with placing tissue expanders in children. ${ }^{3,6,8}$ Some surgeons even wait until patients reach at least 18 years of age, defining an age of maturity, to establish a solid decision-making capacity. ${ }^{9}$ However, at age 18 the degree of physical and psychological maturity varies, as with other chronological ages. More recently, authors have advocated for earlier correction of this deformity, stating that the negative psychological impact of postponing surgery is too great to delay intervention, ${ }^{2}$ acknowledging that possible revision surgery may be ultimately necessary. $^{6}$

The treatment of unilateral breast hypoplasia is generally augmentation of the affected breast. The choice of material for augmentation may be prosthesis-based or autologous. Advantages of implant-based reconstruction include an easier initial operation without compromising the ability to perform autologous tissue transfer at a later date. Therefore, it may be used as a bridge to allow maturation of the patient while concurrently correcting the deformity. ${ }^{7}$ One potential drawback remains the difficulty to achieve upper- and lower-pole symmetry with the contralateral breast due to the lack of plasticity of the implant. In addition, the possibility of capsular contracture and implant rupture, and the eventual need for reoperation should not be overlooked in the adolescent population. ${ }^{7}$ Alternatively, autologous tissue reconstruction will be more stable in the long-term, albeit requiring a longer and more complex operation, a lengthier recovery, and additional donor site morbidity. Regardless of which option is chosen, the contralateral breast may require an intervention to achieve symmetry. This may be in the form of a mastopexy, augmentation, or a combination of both. ${ }^{7}$

Implant-based augmentation mammoplasty is one technique to correct the hypoplastic adolescent breast. It is imperative that these young patients and their families appreciate the potential risks associated with the procedure, including scarring, lactational dysfunction, loss of sensation of the nipple areolar complex, and potential difficulty in tumor detection later in life when breast cancer screening is initiated. ${ }^{9-11}$ Additionally, they need to be aware of the complications associated with the insertion of a prosthetic device, even if the placement is only temporary. In the United States, the selection of breast implants remains limited for adolescent girls and young women. According to U.S. Food and Drug Administration (FDA) regulations, saline breast implants for augmentation mammoplasty are available to women 18 years and older; silicone implants used for the same purpose are approved in women aged 22 years or older. $^{12}$ Both types of implants are available to women of any age for breast reconstruction.

In cases of severe hypoplasia of one or both breasts, strong consideration should be given to performing a two-stage procedure involving prior expansion of the overlying skin envelope. This achieves more natural breast ptosis and lowers the nipple-areolar complex, regardless of what type of reconstruction is ultimately chosen for the patient. $2,6,13,14$ According to Caouette-Laberge and Bortoluzzi, the need for skin expansion should be based on three factors: (1) the severity of hypoplasia, (2) the tightness of the skin envelope, and (3) the location of the areola; any of which may be indication for a two-stage procedure. Furthermore, if autologous reconstruction is performed later, tissue expansion may also reduce the risk of compartment syndrome and subsequent vascular compromise occurring with postoperative swelling of the transferred tissue. ${ }^{7}$

Alternatively, autologous tissue can be used to augment the hypoplastic breast. The choice of flaps differs in adolescents as compared with the adult population due to differences in body habitus during pubertal growth. A paucity of abdominal fat in this age group often makes deep inferior epigastric perforator flaps inadequate in tissue volume for adequate augmentation. ${ }^{7,15}$ In addition, the risk of abdominal wall herniation from future pregnancies is of important concern when nonperforator abdominal flaps are used. ${ }^{15}$ Another option is the pedicled musculocutaneous latissimus dorsi flap, which has the added benefit of avoiding a microsurgical anastomosis. It can be used in combination with other techniques of augmentation, either implant-based or autologous. ${ }^{7,16}$ Alternatively, the superior and inferior gluteal perforator flaps can be used, with the increasing popularity of the latter due to the donor scar location. 7,17,18 Inferior gluteal musculocutaneous flaps in this population generally have low donor-site morbidity, with minimal buttock asymmetry and no functional loss. However, some patients have reported experiencing posterior thigh hypoesthesia following surgery. This may present despite intended preservation of the posterior femoral cutaneous nerve. ${ }^{15}$

Regardless of which reconstructive method is utilized to correct adolescent breast hypoplasia, correction of this deformity remains an ongoing surgical challenge. The timing of intervention is perhaps the most controversial element. Finding the balance between a mature decision-making capacity of the patient and the support of her family is 
essential. Anticipating further breast development during puberty and risking detrimental psychological impact of delaying treatment should be given careful consideration. The patient, her family, and the treating surgeon must carefully weigh these key elements.

\section{Tuberous Breast Deformity}

Tuberous breast deformity was first described in 1976 by Rees and Aston, coined after its resemblance in shape to a tuberous plant root, ${ }^{19}$ and remains one of the most challenging breast anomalies to treat in the developing adolescent (-Fig. 2). In the same year, Vicchione published his experience with the deformity, in describing the recontouring of the domed nipple. ${ }^{20}$ Multiple other terms have been used to describe the tuberous breast, including herniated areolar complex, ${ }^{21}$ tubular breast, ${ }^{22}$ narrow-based breast, ${ }^{23}$ snoopy deformity, ${ }^{24}$ and lower-pole hypoplastic breast. ${ }^{25}$ However, tuberous breast remains the most widely used nomenclature to describe the pathologic condition of glandular hypoplasia with a deficiency in the circumferential skin envelope of the breast base. $^{26}$ It has additionally been characterized by other features, including breast herniation into the areolar region and malposition of the inframammary fold. ${ }^{27}$

Although it has been established that tuberous breast deformity is a rare anomaly of breast development, its incidence is not known. ${ }^{28}$ There is no evidence to suggest familial incidence, nor has it been established that the deformity is secondary to in utero toxin exposure. Early on, one author postulated that the similarity of the breast shape to an animal teat suggests that this is a result of a phylogenetic relapse; however, this concept has not been widely adopted. Several studies have shown that the anomaly occurs bilaterally in 59 to $91 \%$ of patients. ${ }^{29-32}$ The breasts are asymmetric in 70 to $100 \%$ of cases. ${ }^{28,33}$

The etiology of the tuberous breast remains unclear, although several authors have proposed developmental sequences to account for the deformity. Grolleau et al attribute the deformity to the strong adherence of the dermis and muscular plane at the level of the fascia superficialis in the lower pole of the breast. This restricts peripheral expansion,

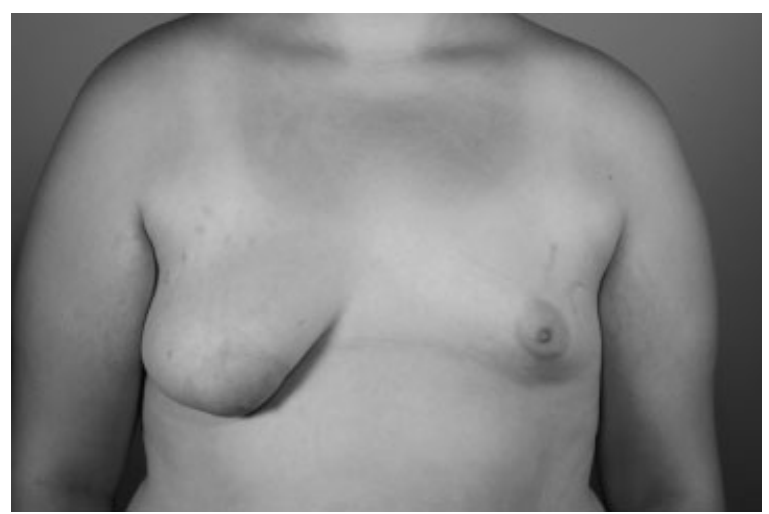

Fig. 2 Right tuberous breast deformity type 1 in an adolescent girl with associated left breast idiopathic hypoplasia. and the breast preferentially protrudes forward creating a tubular shape and an enlarged areola. They propose that this pathological process begins in puberty because no anomaly is apparent prior to the beginning of adolescent breast development. $^{33}$ Mandrekas et al put forward that the tuberous breast deformity results from a constricting fibrous ring peripheral to the nipple-areolar complex that does not allow the expansion of breast parenchymal during the growth phase in adolescence. This prevents the breast from growing inferiorly, encouraging herniation toward the nipple-areola complex. The degree of deformity depends on the degree of constriction of the fibrous band leading to a range of breast pathology, from minor underdevelopment of the inferior medial breast quadrant to four quadrant hypoplasia. ${ }^{30}$

Due to the variability in presentation of patients with this condition, Dinner et al have proposed five key features of the tuberous breast, including the likelihood of each being present. These features are a (1) skin envelope deficiency, (2) hypoplasia of the breast, (3) reduced vertical extent, (4) ptosis, and (5) hypertrophy of the areola. The authors state that the skin envelope deficiency located at the base of the breast is always present; it manifests as a narrow transverse diameter or circumferential constriction. This results in the appearance of breast herniation into the areola; however, no true herniation exists. Second, hypoplasia of the breast is usually present, which is more pronounced at the site of skin constriction. Treatment must address this feature, as the volume of tissue will not correct upon release of the skin envelope. Third, Dinner et al note that breast hypoplasia and skin envelope deficiency lead to a decrease in the overall height of the breast, which translates into a higher breast with exaggerated ptosis. Finally, hypertrophy of the areola is present as compensation from the area of constriction at the base of the breast. ${ }^{34}$

Due to variable severity of the deformity, several classification systems have been proposed over the years. Similar characteristics of these systems allow authors to identify key features that should be addressed for corrective treatment of tuberous breast deformity. Some authors have argued that it may help to resolve nomenclature of the deformity. ${ }^{29}$ The earliest published classification was that of Aston and Rees in 1976. They described two anatomic types of tuberous breast deformity. The first included a breast that has proportional vertical and horizontal deficiencies, a small tuberous-shaped breast, and a large nipple-areola complex. The second consisted of a breast more deficient in the vertical dimension, ptosis, and downward pointing nipple. ${ }^{19}$

Rees' classification remained as the only system until 1996, when von Heimburg et al classified tuberous breast deformity into four types based primarily on degree of breast hypoplasia and deficiency of skin in the subareola (-Fig. 3). Type 1 manifests as hypoplasia of the lower medial quadrant, type II as hypoplasia of the lower medial and lateral quadrants with sufficient skin in the subareolar region, type III as hypoplasia of the lower medial and lateral quadrants, deficiency of skin in the subareolar region, and type IV as severe breast constriction with minimal breast base. Treatment was tailored to the type of breast deformity. ${ }^{29}$ von Heimburg and colleagues 


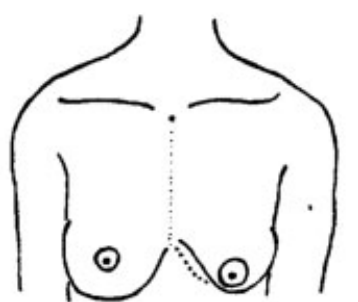

Iype I

hypoplasia of the lower medial quadrant

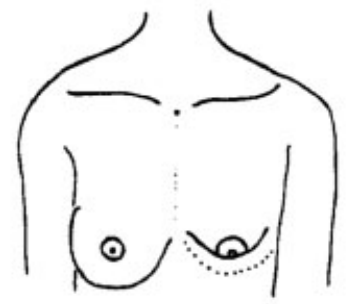

Type III

hypoplasia of the lower medial and lateral quadrant

skin deficiency in the subareolar region

Fig. 3 Classification of tuberous breast deformity (von Heimburg et al) (Reprinted with permission from von Heimburg D, Exner K, Kruft S, Lemperle $G$. The tuberous breast deformity: classification and treatment. Brit J Plast Surg 1996;49(6):339-345).

further refined their classification in 2000 to include profile views of their diagrammatic classification (-Fig. 4). ${ }^{35}$

In 1999, Grolleau et al published a modified version of von Heimburg's classification (- Fig. 5).$^{33}$ They used only three of

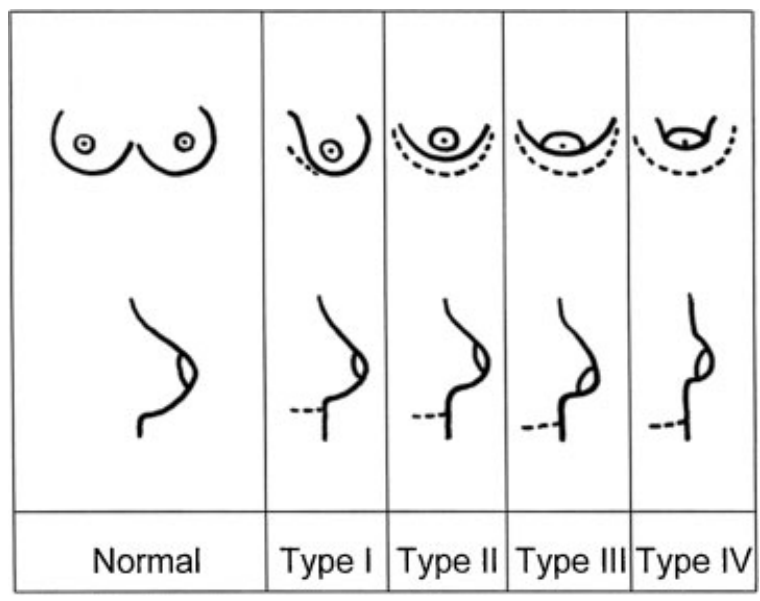

Fig. 4 Revised tuberous breast deformity classification (von Heimburg) (Reprinted with permission from von Heimburg D. Refined version of the tuberous breast classification. Plast Reconstr Surg 2000;105(6):2269-2270).
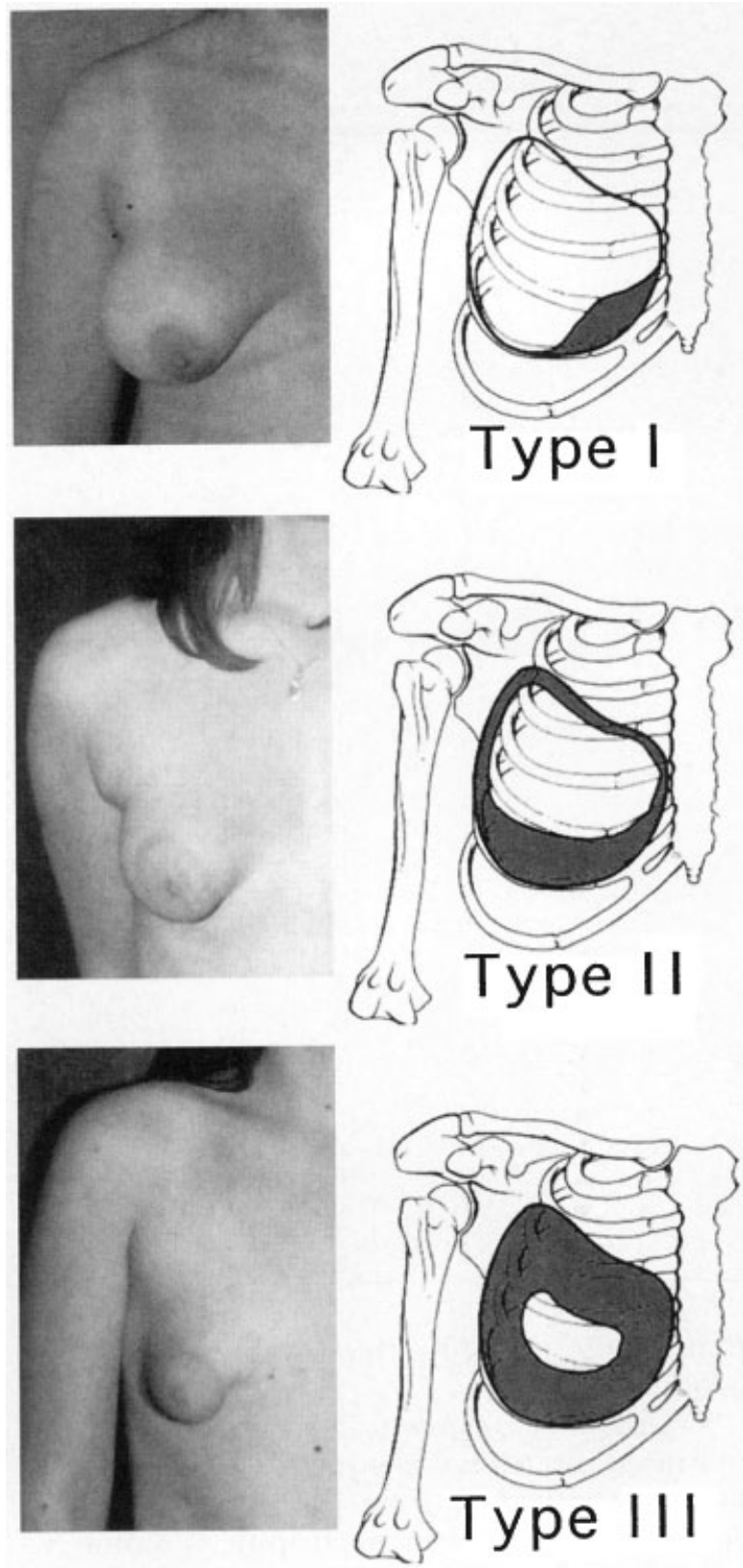

Fig. 5 Tuberous breast deformity classification (Grolleau et al) (Reprinted with permission from Grolleau JL, Lanfrey E, Lavigne B, Chavoin JP, Costagliola M. Breast base anomalies: treatment strategy for tuberous breasts, minor deformities, and asymmetry. Plast Reconstr Surg 1999;104(7):2040-2048).

the four original types, contending that the difference between type 2 and type 3 tuberous breast deformity is clinically insignificant. In normal circumstances, the breast extends between the second and sixth costal cartilage and is divided into four quadrants. Type 1 deformity consists of the lower medial quadrant being deficient and the lateral portion oversized in comparison. In a type 2 deformity, both lower quadrants are deficient causing the areola to point downwards. Type 3 deformity consists of a deficiency in all four quadrants with a narrow base in both vertical and horizontal directions. ${ }^{33}$ Meara et al further modified this classification 
system to include consistent details in each deformity type about the base, inframammary fold, skin envelope, breast volume, and ptosis (-Table $\mathbf{1}$ ). ${ }^{27}$ They specifically exclude characteristics of the areola, maintaining von Heimburg's observation in his 1996 publication that areolar dysmorphology increased in frequency rather than in severity. Therefore, the authors state this feature should not be included in the classification system. $^{27}$

The surgical treatment of tuberous breast deformity in adolescents depends on the severity and classification of the defect, as well as patient perceptions and expectations. Applicable procedures can be divided into operations that involve augmentation, mastopexy, combined augmentation/ mastopexy, and tissue expansion followed by augmentation. ${ }^{27}$ According to Meara et al, the principles of surgical treatment are (1) expansion of the constricted base, (2) lowering of the inframammary fold, (3) increase in the skin envelope when necessary, (4) augmentation of breast volume when necessary, and (5) correction of herniated subareolar breast tissue/correction of areolar size when appropriate. ${ }^{27}$ Various authors have addressed these treatment principles differently.

Rees and Aston were the first to describe management of the constricted breast base in 1976, identifying that breast tissue in this area needs to be released. Since then, several different techniques have been described to expand the breast base, which can be organized into posterior approaches, anterior approaches, and rearrangement with parenchymal flaps. The original description by Rees and Aston was a posterior approach through an inframammary incision, with radial parenchymal incisions designed to expand the base. Meara et al suggest a similar technique; however, they propose that as the severity of the defect increases, breast tissue release at the base is insufficient to correct the defect and an implant is necessary for additional support. Based on their classification, they suggested that type 1 defects can be corrected with radial incisions, type 2 defects require an implant for correction, while type 3 requires tissue expansion prior to exchange with a permanent implant. ${ }^{27}$ Alternatively, Bostwick describes an anterior approach using radial incisions along the inferior half of the breast. ${ }^{36}$ Dinner et al similarly favor this approach; however, they use a submammary flap, including skin, subcutaneous tissue, and breast parenchyma to create a vertical full-thickness release of the constricted base rather than a release of only the breast parenchyma. ${ }^{34}$ The most widely described technique to widen the breast base uses parenchymal flaps. These are designed to fulfill two goals: (1) to expand the constrictive base, and (2) to redistribute volume along the inferior aspect of the breast. In a series of 11 patients, Mandrekas et al describe a periareolar approach, where division of the constricting ring rearranges the inferior part of the breast parenchyma. This creates two breast pillars that are subsequently redraped to add breast volume in the inferior quadrants. In cases of significant volume deficiency, a breast implant is placed. ${ }^{30}$ Other glandular flap techniques to widen the breast base include lateral deepithelialized dermoparenchymal flaps by Golleau et $\mathrm{al}^{33}$ a challenging unfurling of the breast paren-

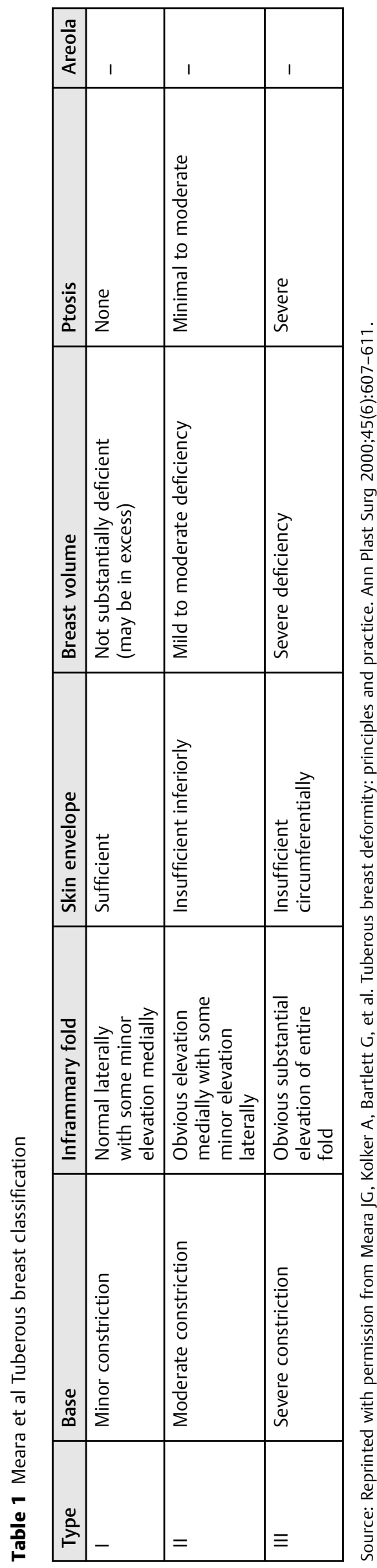


chyma to create an anterior-based pedicle flap by Puckett et $\mathrm{al},{ }^{23}$ and a parenchymal flap with a posterior based pedicle by Ribeiro et al. ${ }^{37}$ This last technique has also been advocated by Grolleau et al in the situation where the breast has sufficient volume and an implant is not necessary. When breast volume is inadequate and an implant is required, the authors support the unfurling technique of Puckett et al because of the high risk of devascularization of the parenchymal flap pedicle that may occur with posterior dissection for implant insertion, even in a retropectoral position. ${ }^{33}$

The second principle of surgically treating tuberous breast deformity is lowering the inframammary fold. Positioning of the new fold is determined by the position of the contralateral nonpathological side. If there is bilateral deformity, the fold is placed at its usual location at the sixth intercostal space. ${ }^{30}$ According to Meara et al's classification, type 1 and 2 deformities can be treated by a subcutaneous dissection in the inferolateral and bilateral inferior quadrants, respectively. In type 3 deformities, they recommend both superior and inferior subcutaneous dissections to create a new skin envelope to correct the substantial vertical deficiency. Regardless of the severity of the deformity, placement of either breast tissue or an implant in the new fold is necessary to maintain its location. ${ }^{27}$ If an implant is used, the inframammary fold should be placed $2 \mathrm{~cm}$ more inferiorly to allow for subsequent contraction. ${ }^{32}$

The adequacy of the skin envelope is critical to correction of tuberous breast deformity. According to Meara et al's classification, only a type 1 breast deformity has a normal skin envelope. There is some debate among authors about whether a skin envelope deficiency indeed exists or whether there is inferior skin laxity with constriction at the level of the subcutaneous tissues. ${ }^{30,31}$ If deemed insufficient, skin envelope deformity can be corrected with locoregional flaps or with tissue expansion. von Heimburg and colleagues have described both Z-plasty and thoracoepigastric flaps as options. ${ }^{29}$ Alternatively, Elliot et al have demonstrated the use of a myocutaneous serratus anterior transposition flap. ${ }^{38}$ Some authors have criticized these techniques due to the scar locations. ${ }^{31}$ Tissue expansion is another method described where the prosthesis is placed centrally to the defect in either a subglandular or subpectoral position. The expander is then used to increase the skin envelope.

When appropriate, redistribution and/or augmentation of the breast volume are important components to correcting the tuberous breast deformity. The decision is dependent on two factors: (1) achieving symmetry with the contralateral breast, and (2) the desires of the patient. According to Meara et al, only type 3 deformities consistently require expansion prior to augmentation, whereas type 2 usually entails augmentation alone. ${ }^{27}$ Some authors, including Ribeiro et al, are opposed to augmentation attributed to preferences of their patient population. ${ }^{37}$ Another area of controversy is the preferred placement of the prosthesis, with some authors advocating subglandular placement ${ }^{19}$ while the majority favor the subpectoral position. ${ }^{31-33,38}$

The final feature of the tuberous breast deformity that needs to be addressed is the correction of any herniated subareolar breast tissue and increased areolar size. Rees et al originally described de-epithelialization of periareolar skin with an outer periareolar incision. Circumferential skin flaps were raised and the de-epithelialized tissue was telescoped inward thus correcting the herniated appearance of the breast and reducing the size of the areola. Meara et al have described a similar technique, ${ }^{27}$ while Atiyeh et al have proposed a perinipple round-block technique that achieves comparable outcomes. ${ }^{39}$

Tuberous breast deformity in adolescents represents a spectrum of pathology. Understanding the features and characteristics at each point in the spectrum allows plastic surgeons to effectively classify the deformity, and therefore optimize aesthetic reconstruction. Since the first description of the tuberous breast in 1976, there have been significant advances in the number of methods used to correct this deformity. As these techniques become more refined and a common classification is adopted, we would benefit from sharing results of larger series of patients to further our understanding of the optimal management options for this condition.

\section{References}

1 Rosen P. Abnormalities of mammary growth and development. In: Rosen's Breast Pathology. Philadelphia, PA: Lippincott Williams \& Wilkins; 2009:23-27

2 Shermak MA. Congenital and developmental abnormalities of the breast. In: Ismail Jatoi, Kaufmann M, eds. Management of Breast Diseases. Heidelberg, Germany: Springer; 2010:37-52

3 Sadove AM, van Aalst JA. Congenital and acquired pediatric breast anomalies: a review of 20 years' experience. Plast Reconstr Surg 2005;115(4):1039-1050

4 Latham K, Fernandez S, Iteld L, Panthaki Z, Armstrong MB, Thaller S. Pediatric breast deformity. J Craniofac Surg 2006;17(3): 454-467

5 Haramis HT, Collins RE. Unilateral breast atrophy. Plast Reconstr Surg 1995;95(5):916-919

6 Oakes MB, Quint EH, Smith YR, Cederna PS. Early, staged reconstruction in young women with severe breast asymmetry.J Pediatr Adolesc Gynecol 2009;22(4):223-228

7 Caouette-Laberge L, Bortoluzzi PA. Correction of breast asymmetry in teenagers. In: Hall-Findlay E, Evans G, eds. Aesthetic and Reconstructive Surgery of the Breast. Philadelphia, PA: Saunders; 2010:601-630

8 Youm T, Margiotta M, Kasabian A, Karp N. Complications of tissue expansion in a public hospital. Ann Plast Surg 1999;42(4):396401, discussion 401-402

9 Jones GE. The patient and the surgeon. In: Bostwick's Plastic and Reconstructive Breast Surgery. St. Louis, MO: Quality Medical Publishing; 2010:3-54

10 Greydanus DE, Matytsina L, Gains M. Breast disorders in children and adolescents. Prim Care 2006;33(2):455-502

11 McGrath MH, Schooler WG. Elective plastic surgical procedures in adolescence. Adolesc Med Clin 2004;15(3):487-502

12 U.S. Food and Drug Administration. Silicone gel-filled breast implants. Silver Spring, MD: U.S. Food and Drug Administration

13 Argenta LC, VanderKolk C, Friedman RJ, Marks M. Refinements in reconstruction of congenital breast deformities. Plast Reconstr Surg 1985;76(1):73-82

14 Pryor LS, Lehman JA Jr, Workman MC. Disorders of the female breast in the pediatric age group. Plast Reconstr Surg 2009;124 (1, Suppl):50e-60e 
15 Dupéré S, Bergeron L, Bortoluzzi P, Del-Duca T, Caouette-Laberge L. Donor-site morbidity of the inferior gluteal musculocutaneous flap for breast reconstruction in teenagers. Ann Plast Surg 2007; 59(6):617-620

16 Rintala AE, Nordström RE. Treatment of severe developmental asymmetry of the female breast. Scand J Plast Reconstr Surg Hand Surg 1989;23(3):231-235

17 Granzow JW, Levine JL, Chiu ES, Allen RJ. Breast reconstruction with gluteal artery perforator flaps. J Plast Reconstr Aesthet Surg 2006;59(6):614-621

18 Boustred AM, Nahai F. Inferior gluteal free flap breast reconstruction. Clin Plast Surg 1998;25(2):275-282

19 Rees TD, Aston SJ. The tuberous breast. Clin Plast Surg 1976; 3(2):339-347

20 Vecchione TR. A method for recontouring the domed nipple. Plast Reconstr Surg 1976;57(1):30-32

21 Bass CB. Herniated areolar complex. Ann Plast Surg 1978;1(4):402-406

22 Williams G, Hoffman S. Mammoplasty for tubular breasts. Aesthetic Plast Surg 1981;5(1):51-56

23 Puckett CL, Concannon MJ. Augmenting the narrow-based breast: the unfurling technique to prevent the double-bubble deformity. Aesthetic Plast Surg 1990;14(1):15-19

24 Gruber RP, Jones HW Jr. The "donut" mastopexy: indications and complications. Plast Reconstr Surg 1980;65(1):34-38

25 Brink RR. Evaluating breast parenchymal maldistribution with regard to mastopexy and augmentation mammaplasty. Plast Reconstr Surg 1990;86(4):715-719, discussion 720-721

26 Versaci AD, Rozzelle AA. Treatment of tuberous breasts utilizing tissue expansion. Aesthetic Plast Surg 1991;15(4):307-312

27 Meara JG, Kolker A, Bartlett G, Theile R, Mutimer K, Holmes AD. Tuberous breast deformity: principles and practice. Ann Plast Surg 2000;45(6):607-611

28 Choupina M, Malheiro E, Pinho C, et al. Tuberous breast: a surgical challenge. Aesthetic Plast Surg 2002;26(1):50-53
29 von Heimburg D, Exner K, Kruft S, Lemperle G. The tuberous breast deformity: classification and treatment. Br J Plast Surg 1996;49(6): 339-345

30 Mandrekas AD, Zambacos GJ, Anastasopoulos A, Hapsas D, Lambrinaki N, Ioannidou-Mouzaka L. Aesthetic reconstruction of the tuberous breast deformity. Plast Reconstr Surg 2003;112(4): 1099-1108, discussion 1109

31 Panettiere P, Del Gaudio GA, Marchetti L, Accorsi D, Del Gaudio A The tuberous breast syndrome. Aesthetic Plast Surg 2000;24(6): 445-449

32 Pardo A, Watier E, Georgieu N, Chevrier S, Pailheret JP. [Tuberous breast syndrome. Report on a series of 22 operated patients]. Ann Chir Plast Esthet 1999;44(6):583-592

33 Grolleau JL, Lanfrey E, Lavigne B, Chavoin JP, Costagliola M. Breast base anomalies: treatment strategy for tuberous breasts, minor deformities, and asymmetry. Plast Reconstr Surg 1999;104(7): 2040-2048

34 Dinner MI, Dowden RV. The tubular/tuberous breast syndrome. Ann Plast Surg 1987;19(5):414-420

35 von Heimburg D. Refined version of the tuberous breast classification. Plast Reconstr Surg 2000;105(6):2269-2270

36 Jones GE. Aesthetic problems and ancillary techniques. In: Bostwick's Plastic and Reconstructive Breast Surgery. St. Louis, MO: Quality Medical Publishing; 2010:755-825

37 Ribeiro L, Canzi W, Buss A Jr, Accorsi A Jr. Tuberous breast: a new approach. Plast Reconstr Surg 1998;101(1):42-50, discussion 51-52

38 Elliott MP. A musculocutaneous transposition flap mammaplasty for correction of the tuberous breast. Ann Plast Surg 1988; 20(2):153-157

39 Atiyeh BS, Hashim HA, El-Douaihy Y, Kayle DI. Perinipple roundblock technique for correction of tuberous/tubular breast deformity. Aesthetic Plast Surg 1998;22(4):284-288 